\title{
Survey of Subjects
}

Adult Education

African History / Local History

African Statistics

Afro-Asiatic Languages \& Literatures

Agriculture / Veterinary Science

Ancient History / Archaeology

Ancient, Medieval, Eastern Philosophy

Animal Husbandry

Applied Physics

Architecture

Archives / Brittle Books

Asian History / Local History

Asian Statistics

Associations, Clubs \& Societies, Misc Associations

Astronomy \& Allied Sciences

Athlethic \& Outdoor Sports \& Games

Atlases, Maps, Charts, Plans

Balto-Slavic Languages \& Literatures

Bible

Bibliography of Belles-Lettres

Bibliography of Geography \& History

Bibliography of Language \& Literature Studies

Bibliography of Philosophy \& Psychology

Bibliography of Religion

Bibliography of Science

Bibliography of Social Sciences

Bibliography of Technology

Bibliography of the Arts

Biological Specimens

Biology

Botanical Sciences

British - Scottish - Irish History / Local History

Buildings

Catholicism / Roman Catholicism

Central European - German History / Local History

Chemical \& Related Technologies

Chemistry \& Allied Sciences

Children's Literature

Children's Non-Fiction

China \& Chinese History / Local History

Christianity / Early Church \& Eastern Churches

Civic \& Landscape Art

Civil / Political Rights

Civil Engineering

Civil Procedure \& Courts

Classical Religions

Collected Works

Commerce / Communications / Transportation

Communities

Comparative Religion

Computer Programming

Computer Science

Constitutional History \& Government

Constitutional Law

Contemporary History

Cooperatives

Criminal Law

\begin{tabular}{|c|c|}
\hline 374 & Criminology \\
\hline 960 & Culture \& Institutions \\
\hline 316 & Current Affairs \\
\hline 470 & Customs / Etiquette / Folklore \\
\hline 630 & Decorative \& Minor Arts \\
\hline 930 & Dictionaries of the Arts \\
\hline 180 & Diseases \\
\hline 636 & Drama \\
\hline 621 & Drawing \& Drawings \\
\hline 720 & Drawing / Decorative \& Minor Arts \\
\hline 025 & Eastern European - Russian History / Local History \\
\hline 950 & Economics \\
\hline 315 & Education \\
\hline 366 & Education of Women \\
\hline 520 & Encyclopedic Works \\
\hline 796 & Engineering \& Allied Operations \\
\hline 912 & English Language \& Literature \\
\hline 460 & Epistemology / Causation / Humankind \\
\hline 220 & Epistles \\
\hline 018 & Essays \\
\hline 019 & Ethics \\
\hline 014 & Ethnology \\
\hline 011 & European History / Local History \\
\hline 012 & European Statistics \\
\hline 015 & Experimental Medicine \\
\hline 013 & Fiction \\
\hline 016 & Financial Economics \\
\hline 017 & Folklore \\
\hline 579 & Forestry / Fruits \\
\hline 574 & French - Monacan History / Local History \\
\hline 580 & From the French Revolution to the First World War \\
\hline 941 & Genealogy / Historical Auxiliary Science \\
\hline 690 & General \& Personal Hygiene \\
\hline 241 & General / Local History of Canada \\
\hline 943 & General / Local History of Mexico, Middle \& South America \\
\hline 660 & General Anthologies of Belles-Lettres \\
\hline 540 & General Bibliography \\
\hline 801 & General Biography \\
\hline 372 & General Collections \\
\hline 951 & General History of the United States \\
\hline 240 & General Language Studies \\
\hline 710 & General Literature Studies \\
\hline 322 & General Nature of Life \\
\hline 624 & General Organizations / Museology \\
\hline 347 & General Periodicals \\
\hline 250 & Generalities \\
\hline 802 & Generalities Dictionaries \\
\hline 380 & Geography \& History Dictionaries \\
\hline 307 & Geography / Travel \\
\hline 230 & Geography / Travel Other Parts \\
\hline 005 & Geography / Travel in Africa \\
\hline 004 & Geography / Travel in Asia \\
\hline 323 & Geography / Travel in Europe \\
\hline 342 & Geography / Travel in North America \\
\hline 934 & Geography / Travel in S \& C America \\
\hline 334 & Germanic Languages \& Literatures \\
\hline 345 & Gospels \& Acts \\
\hline
\end{tabular}

364

306

321

390

745

057

616

820

741

740

947

330

370

376

030

620

420

120

227

840

170

396

940

314

619

830

332

391

634

944

933

929

613

971

972

800

010

920

080

975

400

410

577

060

073

000

050

059

910

919

916

915

914

917

918

430

226 
Graphic Arts / Printmaking and Prints

Gynecology / Obstetrics / Pediatrics / Geriatrics

Higher Education

History

History / Local History of Oceania

History / Local History of Other Areas \& Worlds

History / Local History of Other Parts of Europe

History / Local History of the Americas

Home Economics \& Family Living

Horticulture / Vegetables

Human Anatomy / Cytology / Tissue Biology

Human Physiology

Human Races

Hunting / Game Keeping / Fishing

Hydraulic Engineering

Insurance

International / Foreign Trade

International Economics

International Law / World Organizations

International Relations

Islam / Koran

Italian History / Local History

Journalism / Publishing / General Newspapers

Judaica

Judaism

Labor Economics

Labour, Public Safety Law

Land Economics

Language Dictionaries

Latin, Greek Languages \& Literatures

Law

Letters

Library \& Information Sciences

Life Sciences

Linguistics \& Literature Dictionaries

Logic

Macroeconomics \& Related Topics

Management and Auxilliary Services

Manufacture of Products for Specific Uses

Manufactures

Manuscripts, Book Rarities, Ephemera

Mathematics

Medicine / Nursing

Metaphysics

Microbes

Microscopy in Biology

Middle Ages

Middle Eastern History / Local History

Military \& Nautical Engineering

Military/Naval Science / Military/Naval History / Military/Naval Organization \& Administration

Mining \& Related Operations

Modern Ages

Modern Western Philosophy

Music

New Testament

Newspapers / Periodicals - British Isles

Newspapers / Periodicals - East Europe

Newspapers / Periodicals - Other Geographical Areas

Newspapers / Periodicals - Scandinavia
Newspapers / Periodicals - United States and Canada

Newspapers / Periodicals - West Europe [excl Scandinavia]

North American Statistics

Northern European - Scandinavian History / Local History

Official Government Documents

Old Testament

Organic Evolution / Genetics

Orthodox / Other Christian Denominations / Sects

Other Branches of Engineering

Other Languages \& Literatures

Other Religions

Painting \& Paintings

Paleontology / Paleozoology

Paranormal Phenomena

Penal \& Related Institutions

Pharmacology / Therapeutics

Philosophy

Philosophy \& Related Disciplines Dictionaries

Photography \& Photographs

Physical Anthropology

Physics

Plant Culture

Plant Injuries, Diseases, Pests

Plastic Arts / Sculpture

Poetry

Political Process/Political Parties

Political Science

Private, Commercial Law

Production Economics

Prose / Narratives / Miscellaneous Writings

Protestant Denominations / Anglicanism / Congregational

Psychology

Public Administration

Public Finance

Public Health \& Related Topics

Public Performances \& Stage Presentations

Pure Sciences

Railroads / Roads / Highways

Recreational \& Performing Arts

Relation of Natural Factors to Social Processes

Religion

Religion Dictionaries

Religions of Indic Origin

Religious Beliefs \& Attitudes

Romance Languages \& Literatures

Sanitary \& Municipal Engineering

Satire \& Humor

Schools and Religion

Science Dictionaries

Sciences of the Earth \& Other Worlds

Secondary Education

Sino-Tibetan, Japanese Languages \& Literatures

Social Interaction

Social Problems \& Services / Associations

Social Processes

Social Science Dictionaries

Social Sciences

Social Stratification / Cultural Ethnology / Ethnography

Socialism \& Related Systems

Sociology
071

074

317

948

324

221

575

243

629

490

290

750

560

130

365

615

100

051

770

573

530

631

632

730

810

325

320

346

338

880

242

150

350

336

614

791

500

625

790

304

200

052

280

210

440

628

870

377

055

550

373

480

302

360

303

053

300

305

335

301 
South American Statistics

South Asian, Indian History / Local History

South East Asian History / Local History

Spanish - Portuguese History / Local History

Special Computer Methods

Specific Philosophical Viewpoints

Specific Social Problems \& Services

Speeches

Statistics

Statistics of Other Parts of the World

Statutes, Regulations
Surgery

617

954 Systems

003

959

946

Tax, Trade, Industrial Law

343

Technology

600

006

140

362

Technology (Applied Science) Dictionaries

056

The Arts

700

Unassigned Belles-Lettres

890

850

310

United States: Local History \& Geography

978

United States: Slavery / Civil War Period

976

319

United States: War with Spain to Present

977

348 Zoological Sciences 
